\title{
Cooking Losses of Minerals in Foods and Its Nutritional Significance
}

\author{
Mieko KIMURA and Yoshinori ITOKAwA \\ Department of Hygiene, Faculty of Medicine, Kyoto University, \\ Sakyo-ku, Kyoto 606, Japan
}

\begin{abstract}
Summary To clarify the cooking losses of minerals (sodium, potassium, phosphorus, calcium, magnesium, iron, zinc, manganese, copper), various food materials were analyzed before and after cooking, and the following results were obtained. (1) The mineral contents of cooked foods in mass cooking were on an average about 60-70 percent of those in raw or uncooked foods. (2) Cooking losses were particularly high in minerals of vegetables. (3) Among various cooking methods, loss of mineral was largest in squeezing after boil and in soaking in water after thin slice, followed by parching, frying and stewing. (4) Cooking losses of minerals in meals cooked in home brought about the similar results as those by the mass cooking procedures. (5) The measures to prevent cooking loss are (a) eating the boiled food with the soup, (b) addition of small amount of salt (about $1 \% \mathrm{NaCl}$ ) in boiling, (c) avoidance of too much boiling, (d) selection of a cooking method causing less mineral loss (stewing, frying or parching).
\end{abstract}

Key Words cooking loss, mineral, sodium, potassium, calcium, magnesium, iron, zinc, copper, manganese

Nutritional importance of mineral in mammals has been known, and currently, some trace elements have been recognized to be of nutritional importance, for example, cardiovascular disease caused magnesium deficiency (1), dermatitis or gustatory decline caused by zinc deficiency (2), anemia caused by copper deficiency (3) etc. These mineral are only obtained by extragenous source, that is from diets. But in Japan, the recommended allowance of mineral intakes are not yet set up except iron and calcium. Reports on mineral intake of Japanese are not found so much (4-10). On the other hand, it is considered that cooking causes much loss of minerals from food stuff as is the case of water soluble vitamins (11-14). Thus all minerals contained in food materials is not available from prepared diets. There is few reports on cooking losses of minerals $(15,16)$, especially on trace elements.

In this report, cooking lossess of minerals ( $\mathrm{Na}, \mathrm{K}, \mathrm{P}, \mathrm{Ca}, \mathrm{Mg}, \mathrm{Fe}, \mathrm{Zn}, \mathrm{Cu}, \mathrm{Mn}$ ) by different cooking methods are demonstrated. 


\section{MATERIALS AND METHODS}

Materials. (I) Mineral loss in mass cooking: Eight meals from primary school lunch and 6 meals from university cafeteria in Kyoto, Japan and materials of these meals before cooking were selected as the sample for mineral analyses of mass cooking.

(II) Mineral loss in home cooking: Five meals were cooked in home on the same menu of the mass cooking. Brosilicate glass panns were used in all experiments to avoid exogenous mineral contamination.

(III) Mineral loss of rice: Three kinds of cooked rice from primary school lunch, 2 kinds of cooked rice from university refectory, 2 kinds of rice cooked in home, half-cooked packed rice, pre cooked rice, and rice soaked in water before boiling were prepared for mineral analyses.

(IV) Mineral loss of pork and spinach cooked by different cooking methods, as shown Table 1.

Determination method of mineral. The materials were homogenized by mixer after addition of double distilled water (about $500-1,000 \mathrm{ml}$ in total volume). A part of the homogenate $(25-50 \mathrm{ml})$ was put into the Kjeldahl flask containing nitric acid and then digested by heating. After half digestion perchloric acid was added, and heated again to make color less solution. $\mathrm{Na}, \mathrm{K}, \mathrm{Ca}, \mathrm{Mg}, \mathrm{Fe}, \mathrm{Zn}, \mathrm{Cu}$ and $\mathrm{Mn}$ concentrations were measured using the atomic absorption spectrophotometer (Shimadzu Co., Kyoto Japan). P was measured by Chen's method (17).

\section{RESULTS AND DISCUSSION}

\section{Mineral loss in mass cooking}

Sodium, potassium, phosphorus, calcium, magnesium, iron, zinc, manganese and copper concentrations of primary school lunch and meals of university cafeteria and materials of these meals before cooking are shown in Table 2.

The ranges of cooking loses in these mineral were $0-85 \%$, and sometimes iron concentration showed higher than pre-cooked value. This phenomena may be caused by contamination from pan used in cooking. Cooking loss was particularly high in minerals of vegetables, and largest in squeezing after boiling and thin slice soaked in water, followed by parching, frying and stewing.

\section{Mineral loss in home cooking}

The cooking losses of minerals in meals cooked in home brought about the similar results as those by the mass cooking procedures as shown in Table 2. These range were $0-71 \%$ and average cooking losses was $24 \%$.

\section{Mineral loss in rice}

The mineral concentrations in several cooked rice for primary school lunch 


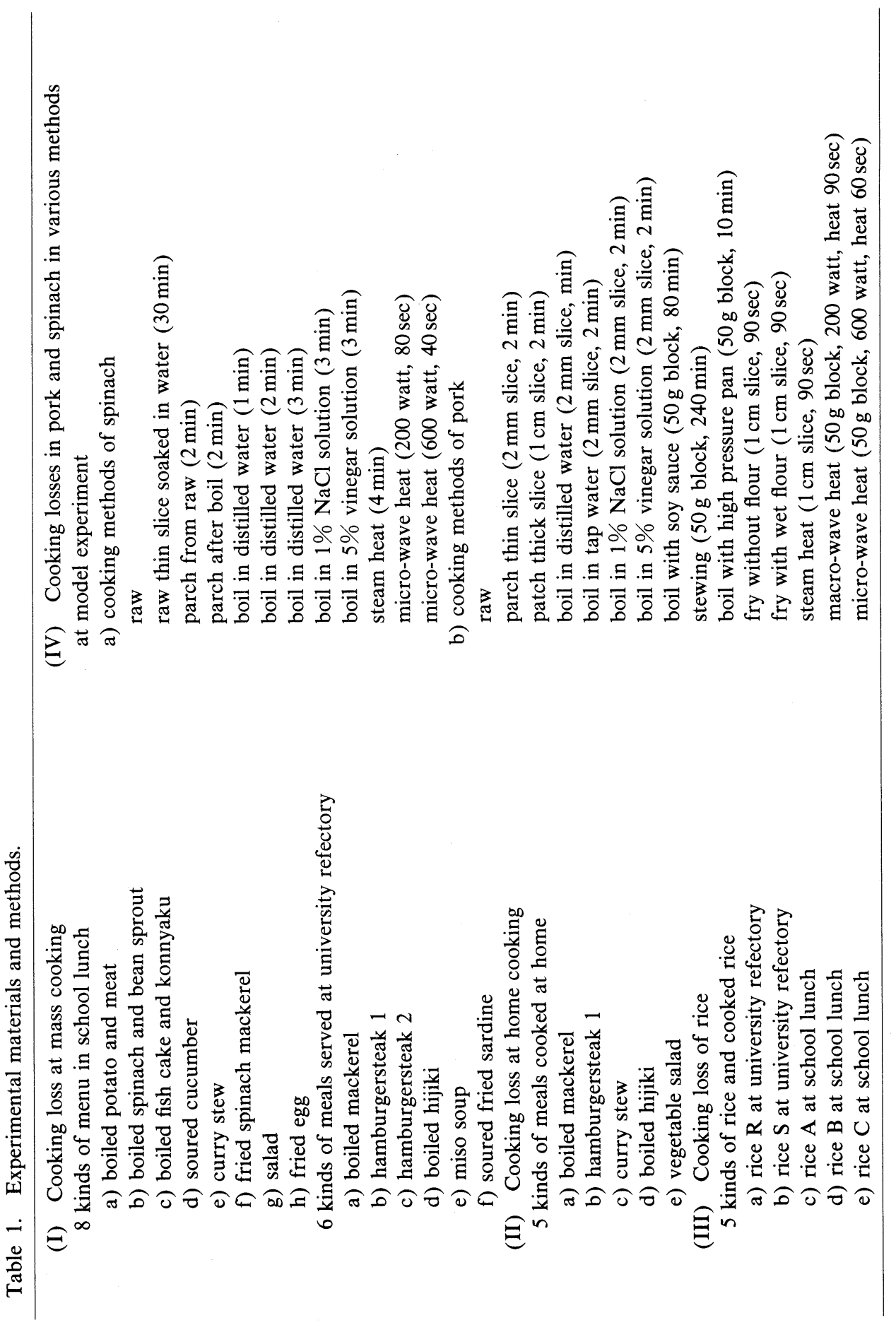

Vol. 36, Suppl. I, 1990 
Table 2. Changes of mineral contents in various menu before and after cooking.

\begin{tabular}{|c|c|c|c|c|c|c|c|c|c|c|c|}
\hline Menu & & $\begin{array}{l}\mathrm{Na} \\
(\mathrm{mg})\end{array}$ & $\begin{array}{c}\mathrm{K} \\
(\mathrm{mg})\end{array}$ & $\begin{array}{c}\mathrm{P} \\
(\mathrm{mg})\end{array}$ & $\begin{array}{l}\mathrm{Ca} \\
(\mathrm{mg})\end{array}$ & $\begin{array}{l}\mathrm{Mg} \\
(\mathrm{mg})\end{array}$ & $\begin{array}{c}\mathrm{Fe} \\
(\mathrm{mg})\end{array}$ & $\begin{array}{c}\mathrm{Zn} \\
(\mathrm{mg})\end{array}$ & $\begin{array}{l}\mathrm{Mn} \\
(\mu \mathrm{g})\end{array}$ & $\begin{array}{c}\mathrm{Cu} \\
(\mu \mathrm{g})\end{array}$ & $\begin{array}{c}\text { Average } \\
(\%)\end{array}$ \\
\hline \multicolumn{12}{|c|}{ Mass cooking process (primary school) } \\
\hline \multirow{2}{*}{$\begin{array}{l}\text { boiled potato \& } \\
\text { meat }\end{array}$} & & 460 & 500 & 79 & 2.3 & 16 & .7 & .8 & 83 & 120 & \\
\hline & loss $(\%)$ & 27 & 2 & 10 & 34 & 20 & 19 & 50 & 64 & 29 & 29 \\
\hline \multirow{2}{*}{$\begin{array}{c}\text { boiled spinach } \& \\
\text { bean sprout }\end{array}$} & & 360 & 140 & 43 & 33 & 24 & .66 & 1.2 & 320 & 100 & \\
\hline & loss $(\%)$ & 22 & 55 & 28 & 8 & 47 & 6 & 8 & 14 & 52 & 27 \\
\hline boiled fish cake \& & & 980 & 230 & 75 & 15 & 15 & .62 & .6 & 240 & 46 & \\
\hline & loss $(\%)$ & 2 & 15 & 23 & 6 & 0 & 6 & 40 & 25 & 4 & 13 \\
\hline \multirow{2}{*}{ soured cucumber } & & 500 & 340 & 6.5 & 10 & 17 & .23 & .41 & 40 & 71 & \\
\hline & loss $(\%)$ & 35 & 39 & 35 & 23 & 60 & 67 & 21 & 69 & 18 & 41 \\
\hline \multirow[t]{2}{*}{ curry stew } & & 980 & 800 & 150 & 43 & 23 & .79 & 2.0 & 400 & 140 & \\
\hline & loss $(\%)$ & 11 & 11 & 6 & 36 & 4 & 10 & 9 & 5 & 18 & 12 \\
\hline \multirow[t]{2}{*}{ fried mackerel } & & 310 & 160 & 100 & 31 & 11 & .28 & .31 & 83 & 48 & \\
\hline & loss $(\%)$ & 45 & 16 & 17 & 11 & 8 & 36 & 21 & 20 & 9 & 21 \\
\hline \multirow[t]{2}{*}{ china style salad } & & 590 & 150 & 24 & 13 & 8.4 & .35 & .40 & 150 & 48 & \\
\hline & loss $(\%)$ & 2 & 27 & 31 & 43 & 14 & 20 & 0 & 17 & 41 & 22 \\
\hline \multirow[t]{2}{*}{ fried egg } & & 360 & 320 & 140 & 13 & 14 & .53 & .53 & 180 & 58 & \\
\hline & loss $(\%)$ & 0 & 11 & 7 & 28 & 22 & 40 & 51 & 49 & 52 & 29 \\
\hline \multicolumn{2}{|c|}{ Average cooking loss $(\%)$} & 18 & 22 & 20 & 24 & 22 & 25 & 25 & 33 & 28 & 24 \\
\hline \multicolumn{12}{|c|}{ Mass cooking process (university refectory) } \\
\hline \multirow[t]{2}{*}{ broiled mackerel } & & 580 & 380 & 190 & 3 & 17 & .59 & .6 & 36 & 72 & \\
\hline & loss $(\%)$ & 8 & 30 & 10 & 79 & 19 & 18 & 40 & 40 & 26 & 32 \\
\hline \multirow{2}{*}{ hamburgersteak 1} & & 1400 & 220 & 220 & 26 & 25 & 1.5 & 2.2 & 350 & 62 & \\
\hline & loss $(\%)$ & 12 & 50 & 4 & 7 & 31 & 17 & 61 & 27 & 69 & 31 \\
\hline \multirow[t]{2}{*}{ hamburgersteak 2} & & 230 & 190 & 82 & 2.4 & 9.6 & 2.0 & 1.8 & 104 & 72 & \\
\hline & loss $(\%)$ & 42 & 24 & 12 & 4 & 4 & -80 & 5 & 4 & 6 & 2 \\
\hline \multirow[t]{2}{*}{ moiled hijiki } & & 800 & 190 & 27 & 65 & 34 & 1.8 & .3 & 403 & 25 & \\
\hline & loss $(\%)$ & 33 & 79 & 67 & 57 & 56 & -50 & 85 & 54 & 74 & 54 \\
\hline \multirow[t]{2}{*}{ miso soup } & & 520 & 86 & 29 & 14 & 8.7 & .85 & .29 & 1301 & 110 & \\
\hline & loss $(\%)$ & 26 & 46 & 49 & 69 & 42 & -165 & 41 & 40 & 8 & 17 \\
\hline \multirow{2}{*}{$\begin{array}{l}\text { soured fried } \\
\text { sardine }\end{array}$} & & 440 & 170 & 100 & 14 & 16 & .47 & .91 & 90 & 74 & \\
\hline & loss $(\%)$ & 33 & 32 & 17 & 7 & 6 & 69 & -75 & 1 & 38 & 14 \\
\hline \multicolumn{2}{|c|}{ Average cooking loss $(\%)$} & 26 & 43 & 26 & 37 & 26 & -32 & 26 & 28 & 37 & 25 \\
\hline
\end{tabular}

and university refectory are determined. Figure 1 shows a part of results.

Cooking losses of magnesium and manganese in rice were largest, followed by phosphorus, copper and potassium. A large part of cooking loss in rice was brought about by soaking in water and washing, in addition of boiling.

But, sodium, calcium and iron contents increased over than pre-cooked rice. Table 3 shows mineral contents of tap water. Sodium, potassium, calcium, magnesium iron and zinc were found, and between them sodium and calcium were 
Table 2. (continued)

\begin{tabular}{|c|c|c|c|c|c|c|c|c|c|c|c|}
\hline Menu & & $\begin{array}{c}\mathrm{Na} \\
(\mathrm{mg})\end{array}$ & $\begin{array}{c}\mathrm{K} \\
(\mathrm{mg})\end{array}$ & $\begin{array}{c}\mathbf{P} \\
(\mathrm{mg})\end{array}$ & $\begin{array}{c}\mathrm{Ca} \\
(\mathrm{mg})\end{array}$ & $\begin{array}{l}\mathrm{Mg} \\
(\mathrm{mg})\end{array}$ & $\begin{array}{c}\mathrm{Fe} \\
(\mathrm{mg})\end{array}$ & $\begin{array}{c}\mathrm{Zn} \\
(\mathrm{mg})\end{array}$ & $\begin{array}{c}\mathrm{Mn} \\
(\mu \mathrm{g})\end{array}$ & $\begin{array}{c}\mathrm{Cu} \\
(\mu \mathrm{g})\end{array}$ & $\begin{array}{c}\text { Average } \\
(\%)\end{array}$ \\
\hline \multicolumn{12}{|c|}{ Home cooking process } \\
\hline \multirow[t]{2}{*}{ broiled mackerel } & & 599 & 298 & 94 & 1.5 & 28 & .58 & 46 & - & 49 & \\
\hline & loss $(\%)$ & 30 & 20 & 38 & 21 & 59 & 2 & 39 & - & 12 & 28 \\
\hline \multirow[t]{2}{*}{ hamburgersteak 1} & & 218 & 87 & 43 & 2.1 & 6.9 & .54 & .76 & - & 32 & \\
\hline & loss $(\%)$ & 54 & 67 & 66 & 47 & 59 & 46 & 71 & - & 41 & 56 \\
\hline \multirow[t]{2}{*}{ curry stew } & & 1030 & 348 & 134 & 42 & 9.8 & .93 & 1.5 & - & 109 & \\
\hline & loss $(\%)$ & 14 & 8 & 6 & 19 & 1 & 22 & 17 & & 5 & 8 \\
\hline \multirow[t]{2}{*}{ boiled hijiki } & & 1037 & 469 & 57 & 60 & 39 & .39 & .14 & - & 43 & \\
\hline & loss $(\%)$ & 19 & 3 & 0 & 9 & 33 & 52 & 61 & - & 4 & 23 \\
\hline \multirow[t]{2}{*}{ salad } & & 215 & 271 & 16 & 215 & 7.3 & .29 & .25 & 79 & 33 & \\
\hline & loss $(\%)$ & 30 & 13 & 16 & 30 & 12 & 17 & 0 & 25 & 49 & 21 \\
\hline Average cooking & loss $(\%)$ & 29 & 5 & 25 & 25 & 33 & 28 & 38 & 25 & 22 & 24 \\
\hline
\end{tabular}

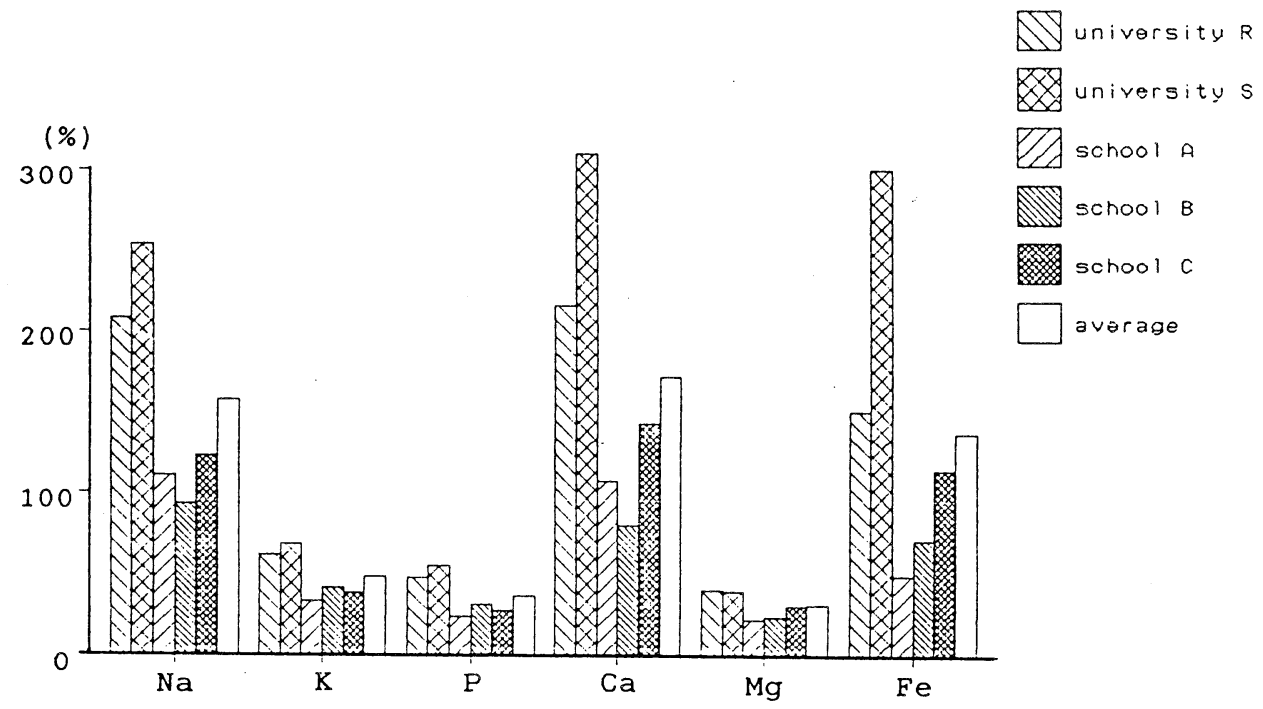

Fig. 1. Remain percentage of mineral content in rice after cooking.

especially high. From this fact, these phenomena may be caused by contamination of tap water or pan used for cooking.

Model experiments for mineral losses of pork and spinach cooked by different cooking methods

Mineral contents of spinach and pork in pre- and post-cooked sample by different cooking methods are shown in Tables 4 and 5 . 
Table 3. Mineral contents of tap water. (ppm)

\begin{tabular}{lrcccccccc}
\hline & $\mathrm{Na}$ & $\mathrm{K}$ & $\mathrm{P}$ & $\mathrm{Ca}$ & $\mathrm{Mg}$ & $\mathrm{Fe}$ & $\mathrm{Zn}$ & $\mathrm{Mn}$ & $\mathrm{Cu}$ \\
\hline tap water A & 9.7 & 1.8 & - & 13.0 & 2.5 & 0.12 & 0.022 & - & - \\
tap water B & 15.0 & 1.6 & - & 2.6 & 3.2 & - & 0.22 & - & - \\
\hline
\end{tabular}

Table 4. Cooking losses of minerals in spinach by various cooking methods.

\begin{tabular}{|c|c|c|c|c|c|c|c|}
\hline \multicolumn{2}{|c|}{ Cooking method } & $\mathrm{Na}$ & $\mathrm{K}$ & $\mathrm{Ca}$ & $\mathrm{Mg}$ & $\mathrm{Fe}$ & $\mathrm{Zn}(\mathrm{mg} / 100 \mathrm{~g})$ \\
\hline \multicolumn{2}{|l|}{ raw } & 16 & 490 & 25 & 35 & 1.7 & 0.23 \\
\hline \multicolumn{2}{|c|}{ raw thin slice dipped } & 5.1 & 255 & 18 & 17 & 0.16 & 0.1 \\
\hline in water & loss $(\%)$ & 68.1 & 48.0 & 28.0 & 51.4 & 90.6 & 56.5 \\
\hline \multicolumn{2}{|l|}{ parch from raw } & 11 & 247 & 19 & 17 & 0.2 & 0.15 \\
\hline & loss $(\%)$ & 31.3 & 49.6 & 24.0 & 51.4 & 88.2 & 34.0 \\
\hline \multirow[t]{2}{*}{ parch after boil } & & 7.6 & 208 & 6.3 & 6.0 & 0.15 & 0.13 \\
\hline & loss $(\%)$ & 52.5 & 57.6 & 74.8 & 82.9 & 91.2 & 43.5 \\
\hline \multirow[t]{2}{*}{ boil (1 min) } & & 7.5 & 208 & 19 & 8.3 & 0.47 & 0.11 \\
\hline & loss $(\%)$ & 53.1 & 57.6 & 24.0 & 76.3 & 72.4 & 52.2 \\
\hline \multirow[t]{2}{*}{ boil (2 min) } & & 4.5 & 137 & 17 & 5.8 & 0.24 & 0.1 \\
\hline & loss $(\%)$ & 71.9 & 72.0 & 32.0 & 83.4 & 85.9 & 56.5 \\
\hline \multirow[t]{2}{*}{ boil (3 min) } & & 6.9 & 135 & 10 & 4.5 & 1.2 & 0.12 \\
\hline & loss $(\%)$ & 56.9 & 72.4 & 60.0 & 87.1 & 29.4 & 47.8 \\
\hline \multirow{2}{*}{$\begin{array}{l}\text { boil in } 1 \% \mathrm{NaCl} \\
\text { (1 } \mathrm{min})\end{array}$} & & 12.0 & 206 & 11.0 & 7.9 & 0.25 & 0.12 \\
\hline & loss $(\%)$ & 25.0 & 58.0 & 56.0 & 77.4 & 85.3 & 47.8 \\
\hline \multicolumn{2}{|c|}{ boil in $5 \% \mathrm{CH}_{3} \mathrm{COOH}$} & 6.6 & 174 & 15 & 4.9 & 0.16 & 0.05 \\
\hline$(1 \mathrm{~min})$ & loss $(\%)$ & 58.8 & 64.5 & 40.0 & 86.0 & 90.6 & 78.3 \\
\hline \multirow[t]{2}{*}{ steam } & & 6.3 & 194 & 13 & 7.6 & 0.11 & 0.16 \\
\hline & loss $(\%)$ & 60.6 & 60.4 & 48.0 & 78.3 & 93.5 & 30.4 \\
\hline \multicolumn{8}{|l|}{ microwave } \\
\hline \multicolumn{2}{|l|}{ slow heat } & 9.1 & 232 & 15 & 7.7 & 1.7 & 0.13 \\
\hline & loss $(\%)$ & 43.1 & 52.7 & 40.0 & 78.0 & 0.0 & 43.5 \\
\hline quick he & & 7.0 & 243 & 10 & 7.9 & 0.88 & 0.19 \\
\hline & loss $(\%)$ & 56.3 & 50.4 & 60.0 & 77.4 & 48.2 & 17.4 \\
\hline
\end{tabular}

Cooking loss of spinach minerals in squeezing after boiling or in thin slice was highest, followed by boiling in tap water, in 5\% sodium acetate, in 1\% salt solution, heating with steam, microwave heating and parching. Cooking losses of minerals in spinach were largest in iron and magnesium, followed by in potassium, sodium, zinc and phosphorus.

Cooking loss of mineral of thin slice pork in boiling was largest, followed by boiling in $1 \%$ acetic acid, heating with steam, stewing, frying without flour, frying with dry flour, boiling in 1\% salt, microwave heating and frying with wet flour. Cooking loss of minerals in pork was largest in zinc, followed by sodium, potassium, calcium, iron, magnesium and phosphorus. In these samples, cooking loss 
Table 5. Cooking losses of minerals in pork by various cooking methods.

\begin{tabular}{|c|c|c|c|c|c|c|c|}
\hline Cooking method & $\mathrm{Na}$ & $\mathrm{K}$ & $\mathbf{P}$ & $\mathrm{Ca}$ & $\mathrm{Mg}$ & $\mathrm{Fe}$ & $\begin{array}{c}\mathrm{Zn} \\
(\mathrm{mg} / 100 \mathrm{~g})\end{array}$ \\
\hline raw & 31 & 638 & 191 & 1.3 & 23 & 1.0 & 4.3 \\
\hline parch thin slice & $\begin{array}{r}14 \\
54.8\end{array}$ & $\begin{array}{r}541 \\
15.2\end{array}$ & $\begin{array}{r}193 \\
-1.0\end{array}$ & $\begin{array}{l}0.98 \\
24.6\end{array}$ & $\begin{array}{r}22 \\
4.3\end{array}$ & $\begin{array}{r}0.99 \\
1.0\end{array}$ & $\begin{array}{r}1.9 \\
55.8\end{array}$ \\
\hline $\begin{array}{l}\text { parch thick slice } \\
\qquad \text { loss }(\%)\end{array}$ & $\begin{array}{r}24 \\
22.6\end{array}$ & $\begin{array}{r}523 \\
18.0\end{array}$ & $\begin{array}{r}178 \\
6.8\end{array}$ & $\begin{array}{r}1.0 \\
23.1\end{array}$ & $\begin{array}{r}22 \\
4.3\end{array}$ & $\begin{array}{l}0.79 \\
21.0\end{array}$ & $\begin{array}{r}1.7 \\
60.5\end{array}$ \\
\hline $\begin{array}{l}\text { boil distilled water } \\
\qquad \text { loss (\%) }\end{array}$ & $\begin{array}{r}8 \\
74.2\end{array}$ & $\begin{array}{r}147 \\
77.0\end{array}$ & $\begin{array}{r}80 \\
58.1\end{array}$ & $\begin{array}{l}0.61 \\
53.1\end{array}$ & $\begin{array}{r}11 \\
52.2\end{array}$ & $\begin{array}{l}0.52 \\
48.0\end{array}$ & $\begin{array}{r}1.1 \\
74.4\end{array}$ \\
\hline boil tap water & $\begin{array}{r}16 \\
48.4\end{array}$ & $\begin{array}{r}270 \\
57.7\end{array}$ & $\begin{array}{r}80 \\
58.1\end{array}$ & $\begin{array}{l}0.63 \\
51.5\end{array}$ & $\begin{array}{r}12 \\
47.8\end{array}$ & $\begin{array}{l}0.78 \\
22.0\end{array}$ & $\begin{array}{r}1.7 \\
60.4\end{array}$ \\
\hline biol in $1 \% \mathrm{NaCl}$ loss $(\%)$ & $\begin{array}{r}49 \\
-58.1\end{array}$ & $\begin{array}{r}324 \\
49.2\end{array}$ & $\begin{array}{r}79 \\
58.6\end{array}$ & $\begin{array}{l}0.71 \\
45.4\end{array}$ & $\begin{array}{r}11 \\
52.2\end{array}$ & $\begin{array}{l}0.62 \\
38.0\end{array}$ & $\begin{array}{r}1.2 \\
72.1\end{array}$ \\
\hline $\begin{array}{l}\text { boil in } 5 \% \mathrm{CH}_{3} \mathrm{COOH} \\
\text { loss }(\%)\end{array}$ & $\begin{array}{r}15 \\
51.6\end{array}$ & $\begin{array}{r}221 \\
65.4\end{array}$ & $\begin{array}{r}149 \\
22.0\end{array}$ & $\begin{array}{l}0.35 \\
73.1\end{array}$ & $\begin{array}{r}9.7 \\
57.8\end{array}$ & $\begin{array}{l}0.33 \\
67.0\end{array}$ & $\begin{array}{l}0.73 \\
83.0\end{array}$ \\
\hline $\begin{array}{l}\text { boil with soy sauce } \\
\qquad \text { loss (\%) }\end{array}$ & $\begin{array}{r}22 \\
29.0\end{array}$ & $\begin{array}{r}387 \\
39.3\end{array}$ & $\begin{array}{r}73 \\
61.8\end{array}$ & $\begin{array}{l}0.63 \\
51.5\end{array}$ & $\begin{array}{r}19 \\
17.4\end{array}$ & $\begin{array}{l}0.53 \\
47.0\end{array}$ & $\begin{array}{r}1.6 \\
62.8\end{array}$ \\
\hline $\begin{array}{l}\text { stewing for long time } \\
\qquad \text { loss }(\%)\end{array}$ & $\begin{array}{r}13 \\
58.1\end{array}$ & $\begin{array}{r}251 \\
60.7\end{array}$ & $\begin{array}{r}129 \\
32.5\end{array}$ & $\begin{array}{r}0.6 \\
53.8\end{array}$ & $\begin{array}{r}7.8 \\
66.1\end{array}$ & $\begin{array}{l}0.66 \\
34.0\end{array}$ & $\begin{array}{l}0.99 \\
77.0\end{array}$ \\
\hline $\begin{array}{l}\text { boil by high pressure } \\
\text { pan loss (\%) }\end{array}$ & $\begin{array}{r}4.9 \\
84.2\end{array}$ & $\begin{array}{r}247 \\
61.3\end{array}$ & $\begin{array}{r}105 \\
45.0\end{array}$ & $\begin{array}{l}0.49 \\
62.3\end{array}$ & $\begin{array}{r}15 \\
34.8\end{array}$ & $\begin{array}{l}0.53 \\
47.0\end{array}$ & $\begin{array}{r}2.1 \\
51.2\end{array}$ \\
\hline $\begin{array}{l}\text { fry without flour } \\
\qquad \operatorname{loss}(\%)\end{array}$ & $\begin{array}{r}7.5 \\
75.8\end{array}$ & $\begin{array}{r}290 \\
54.5\end{array}$ & $\begin{array}{r}189 \\
1.0\end{array}$ & $\begin{array}{l}0.61 \\
53.1\end{array}$ & $\begin{array}{r}13 \\
43.5\end{array}$ & $\begin{array}{l}0.39 \\
61.0\end{array}$ & $\begin{array}{l}0.68 \\
84.2\end{array}$ \\
\hline fry with flour & $\begin{array}{r}9.3 \\
70.0\end{array}$ & $\begin{array}{r}251 \\
60.7\end{array}$ & $\begin{array}{r}219 \\
-14.7\end{array}$ & $\begin{array}{l}0.96 \\
26.2\end{array}$ & $\begin{array}{r}23 \\
0.0\end{array}$ & $\begin{array}{r}0.5 \\
50.0\end{array}$ & $\begin{array}{r}3.6 \\
16.3\end{array}$ \\
\hline $\begin{array}{l}\text { fry with wet flour } \\
\qquad \operatorname{loss}(\%)\end{array}$ & $\begin{array}{r}16 \\
48.4\end{array}$ & $\begin{array}{r}734 \\
-15.0\end{array}$ & $\begin{array}{r}92 \\
51.8\end{array}$ & $\begin{array}{r}1.0 \\
23.1\end{array}$ & $\begin{array}{r}26.0 \\
-13.0\end{array}$ & $\begin{array}{l}0.63 \\
37.0\end{array}$ & $\begin{array}{r}4.4 \\
-2.3\end{array}$ \\
\hline loss $(\%)$ & $\begin{array}{r}27 \\
12.9\end{array}$ & $\begin{array}{r}210 \\
67.1\end{array}$ & $\begin{array}{r}184 \\
3.7\end{array}$ & $\begin{array}{l}0.43 \\
66.9\end{array}$ & $\begin{array}{r}11 \\
52.2\end{array}$ & $\begin{array}{l}0.66 \\
34.0\end{array}$ & $\begin{array}{r}1.2 \\
72.1\end{array}$ \\
\hline microwave & & & & & & & \\
\hline $\begin{array}{l}\text { slow heat } \\
\text { loss }(\%)\end{array}$ & $\begin{array}{r}11 \\
64.5\end{array}$ & $\begin{array}{r}506 \\
20.7\end{array}$ & $\begin{array}{r}135 \\
29.3\end{array}$ & $\begin{array}{r}1.1 \\
15.4\end{array}$ & $\begin{array}{r}19 \\
17.4\end{array}$ & $\begin{array}{r}0.95 \\
5.0\end{array}$ & $\begin{array}{r}2.3 \\
46.5\end{array}$ \\
\hline quick heat & 8.9 & 376 & 180 & 0.78 & 14 & 1.0 & 2.2 \\
\hline loss $(\%)$ & 71.3 & 41.1 & 5.8 & 40.0 & 39.1 & 0.0 & 48.8 \\
\hline
\end{tabular}

was dependent on the type of cooking method irrespective of the type of mineral. Cooking loss of minerals is suspected to be caused by outflow of minerals from food materials.

The measures to prevent cooking loss of minerals are (a) eating the boiled food with the soup, (b) addition of small amount of salt (about $1 \% \mathrm{NaCl}$ ) in boiling, (c) avoidance of too much boiling, and (d) selection of a cooking method causing less mineral loss (stewing, frying or parching). 


\section{REFERENCES}

1) Seeling, M. S., and Haggivei, H. A. (1974): Magnesium interrelationships in ischemic heart disease. Am. J. Clin. Nutr., 27, 59-79.

2) Kay, R. G., Tasman-Jones, C., Pybus, J., Whiting, R., and Black, H. (1987): A syndrome of acute zinc deficiency during total parental alimentation in man. Ann. Surg., 183, 33-42.

3) Graham, G. G., and Cordano, A. (1969): Cooper depletion and deficiency in the marnourished infant. Johns Hopkins Med. J., 124, 139-150.

4) Kimura, M., and Itokawa, Y. (1989): Food habits and magnesium intake of Japanese. Magnesium in Health and Disease, John Libbey \& Co. Ltd., 63-69.

5) Kimura, M., Kimura, I., Nagai, K., Morikawa, M., Yasunaga, T., Natuyama, S., and Itokawa, Y. (1984): Estimation of magnesium intake of Japanese. Magnesium, 3, 15.

6) Kimura, M., Nagai, K., Yasunaga, T., Natuyama, S., Kimura, I., and Itokawa, Y. (1984): A survey study on mineral intake of Japanese. Proceedings The First Symposium on Trace Element Res., 71-82.

7) Kimura, M., Nagai, K., and Itokawa, Y. (1985): Estimation of magnesium and other mineral intake of Japanese by survey study. J. Am. Coll. Nutr., 4, 374.

8) Teraoka, H., Morii, F., and Kobayashi, J. (1981): The concentrations of 24 elements in foodstuffs and the estimate of their daily intake. J. Jpn. Soc. Food Nutr., 34, 221239.

9) Suzuki, T., Kobayashi, K., Yamauchi, Y., Hongo, T., Ishida, H., and Suzuki, H. (1987): Dietary intake of zinc: Comparison between the analysed value and the calculated value. Soc. Food Nutr., 40, 179-192.

10) Morgan, K. J., Stampley, G. L., Zabik, M. E., and Fisher, D. R. (1985): Magnesium and calcium intakes of the U.S. population. J. Am. Coll. Nutr., 4, 195-206.

11) Kimura, M., Saito, N., and Itokawa, Y. (1982): Cooking loss of vitamin B1 in foods and its nutritional significance. Vitamins (J. Vitamin. Soc. Jpn.), 56, 415-423.

12) Somogyi, J. C. (1945): Versuche über Wirkstoffe, die den Ascorbinsaureabbau hemmen. Z. Vitaminforsch., 16, 134-168.

13) Ariyama, T. (1948): Studies on the decay of thiamine in the processes of storage, processing and cooking of foods. Vitamins (J. Vitamin. Soc. Jpn.), 1, 51-56.

14) Yagi, N., and Itokawa, Y. (1979): Cleavage of thiamine by chlorine in tap water. $J$. Nutr. Sci. Vitaminol., 25, 281-287.

15) Oh, C. K., Lucker, P. W., Wetzelsberger, N., and Kuhlmann, F. (1986): The determination of magnesium, calcium, sodium in assorted foods with special attention to the loss of electrolytes after various forms of foods preparations. Mag.-Bull., 8, 297-302.

16) Yakushiji, I., and Kagawa, Y. (1975): Changes in potassium contents of therapeutical diets in nephropathy by cooking methods. J. Jap. Soc. Food Nutr., 28, 67-77.

17) Chen, P. S., Jr., Toribata, T. Y., and Warner, H. (1956): Microdetermination of phosphorus. Anal. Chem., 28, 1756. 


\section{DISCUSSION-CONTRIBUTION}

Speaker: Kimura, M.

Question: Müller, H. R.

Mineral contents of studied meals and foods show great variability from $<100$ to $>200 \%$. What is considered $100 \%$ level?

Answer: The increase of sodium, calcium, and iron in cooked foods may be caused by contamination from mineral of tap water or pan used in cooking.

Speaker: Kimura, M.

Question: Puwastien, P. (Thailand)

How do you express your results before you calculate the losses due to processing? Should it be on dry weight basis? Wet weight basis? I think we should consider the values on dry wet basis. Because moisture changes the values greatly. And I do not think contamination from water can change the values so much.

Answer: We usually calculate on wet weight basis and there is no problem usually. We used wet digestion method with nitric acid and perchloric acid usually for mineral determination. 